\title{
Cognitive Load in Solving Mathematics Problems: Validating the Role of Motivation and the Interaction Among Prior Knowledge, Worked Examples, and Task Difficulty
}

\author{
Udita Gupta ${ }^{1}$, Robert Z. Zheng ${ }^{1 *}$
}

\section{University of Utah, USA}

*Corresponding Author: robert.zheng@utah.edu

Citation: Gupta, U. and Zheng, R. Z. (2020). Cognitive Load in Solving Mathematics Problems: Validating the Role of Motivation and the Interaction Among Prior Knowledge, Worked Examples, and Task Difficulty. European Journal of STEM Education, 5(1), 05. https://doi.org/10.20897/ jsteme/9252

Published: November 24, 2020

\begin{abstract}
Cognitive load can play a key role in learners' abilities to solve complex problems like mathematics. Many factors can affect the presence of cognitive load in learning including instructional strategy, task difficulty and prior knowledge. To understand the interaction of above factors and their influence on learner cognitive load and performance, a three-way interaction study was conducted with worked example (full- vs. completion-worked examples), task difficulty (easy vs. difficult) and prior knowledge (high vs. low) serving as independent variables, and cognitive load and performance as dependent variables. One hundred and sixty participants were recruited who were randomly assigned into one of eight learning conditions. Results revealed a significant three-way interaction by posttest. It was found that higher-prior knowledge learners performed better with completion-worked examples than full-worked examples whereas lower-prior knowledge learners performed better with full-worked examples than completion-worked examples. Significant positive correlation was found between intrinsic and germane cognitive load implying that interest in the instructional domain is an important determinant in effecting germane cognitive load.
\end{abstract}

Keywords: cognitive load, math education, motivation, prior knowledge, worked examples

\section{INTRODUCTION}

It is widely recognized that learners' performance in complex learning like mathematics and STEM related domains can be significantly influenced by the cognitive load during learning (Ayres, 2018; Sweller \& Chandler, 1991). Further, the amount of cognitive load and learner performance are found to be related to factors like domain prior knowledge (Kalyuga, Chandler, \& Sweller, 1998; Richter \& Scheiter, 2019), task difficulty (Lee, Plass, \& Homer, 2006; Lynch, Hurley, \& Cumiskey, 2019), and instructional strategy (Saw, 2017; Wallen, Plass, \& Brunken, 2005). Despite the individual correlations between cognitive load and the above factors, the question of how these factors together function to affect the degree of cognitive load presence and performance remains unanswered. It is argued that factors like domain prior knowledge, task difficulty, and instructional strategy may concurrently influence and mediate the functional role of cognitive load in learning (Gupta, 2015). As such, studying how these factors interact to impact cognitive load may help reveal the roles of key individual and educational variables on learners' outcomes in learning, particularly in STEM learning. The first goal of present study is therefore to explore the interaction among the factors previously mentioned in terms of their influence on cognitive load and learner performance. 
There is ample evidence indicating the correlation between learners' interest and performance in complex learning (Hosbein \& Barbera, 2020). It is found that learners who are motivated are more likely to engage in deeplevel thinking (Liu, Toprac, \& Yuen, 2009; O’Conner \& Domingo, 2020). Evidence from empirical research further suggests that the status of cognitive load and mental effort investment are closely associated with learner's motivation (Plass \& Kalyguga, 2019; Schnotz, 2010). Learners who are cognitively overloaded often experience frustration and are less motivated. In contrast, learners with low cognitive load are more likely to avail themselves of precious cognitive resources in working memory to engage in meaningful learning and become motivated. Regardless, the research on motivation and cognitive load is underexplored, particularly the relationship between types of cognitive load and motivation in the context of prior knowledge, instructional strategy and task difficulty interaction. Thus, the second goal of this study is to examine the relationship between types of cognitive load and motivation in a STEM related domain.

\section{Cognitive Load Theory (CLT)}

Working memory poses significant constraints on human learning due to its limitations in processing capacity and duration (Baddeley, 1990; Baddeley \& Hitch, 1974; Zheng \& Gardner, 2020). Studies have also shown that working memory processing capacity is closely associated with the availability of its cognitive resources (Cook, Zheng, \& Blaz, 2009; Smith et al., 2019). Smith et al. (2019) find that by activating learners' schemas in domain areas, the learners experience lower cognitive load as they are able to retrieve information in chunks, which in turn makes cognitive resources available in working memory for learning. Smith et al.'s findings consist with the literature showing a strong correlation between cognitive resources, cognitive load and performance. Similar findings were obtained by Fuchs, Fuchs and Seethaler (2020) who confirmed the relationship between cognitive resources and working memory capacity in mathematics problem solving.

However, high cognitive load does not necessarily end up in low performance and low cognitive load does not mean learners will always perform well in learning. In fact, the functional role of cognitive load is determined by its relevance to learning (Sweller, 2010; Sweller, Van Merriënboer, \& Paas, 1998). According to Sweller (2018), there are different types of cognitive load and they differ from each other depending on their relevance to learning. Cognitive load relevant to learning facilitates the construction of knowledge and schemata whereas irrelevant cognitive load can be detrimental to learning resulting in the reduction of cognitive resources in working memory. The following discussion focuses on the types of cognitive load and their relevance to learning.

Three types of cognitive load exist in learning. They are: intrinsic, extraneous and germane cognitive load. Intrinsic cognitive load is defined by the difficulty of the instructional materials that cannot be changed or altered. Extraneous cognitive load refers to the mental load caused by improper instructional design like imposing redundant information in learning materials that requires learners' additional processing effort. The extraneous cognitive load is irrelevant to learning and therefore should be eliminated. The last type of cognitive load is germane cognitive load. It is the mental load induced by the efforts to construct new knowledge or build new schema (Sweller et al., 1998). As such, it is relevant to learning and should be optimized to support learning. According to Sweller (2010; 2018), the three types of cognitive load are additive and they together cannot exceed the total working memory capacity. Therefore, in any instructional situation, intrinsic and extraneous cognitive load cannot be both high at the same time. In other words, if intrinsic cognitive load is high, extraneous cognitive load must be low to allow working memory to have sufficient cognitive resources to process the information. On the other hand, if extraneous cognitive load is high, intrinsic cognitive load must be low so the learner is able to deal with additional mental load caused by the improper design of instruction. The negative relationship between intrinsic and extraneous cognitive load also applies to the relationship between germane cognitive load and extraneous cognitive load. That is, the higher the extraneous cognitive load is, the fewer cognitive resources become available in working memory, and the lower the germane cognitive load will be.

The relationship between germane and intrinsic cognitive load reflects a state of cognitive resource distribution in working memory. For germane cognitive load to occur, the content must be difficult enough so the learner experiences cognitive pressure at some level to become motivated to learn. Overly difficult material will impose high intrinsic cognitive load on the learner thus depleting his/her cognitive resources in working memory. The design of instruction should thus follow what Vygotsky (1978) described as the zone of proximal development (ZPD) where the content is challenging enough but not cognitively overwhelming, which "induces learners' germane cognitive load to engage in meaningful and sustained effort in knowledge acquisition" (Zheng \& Gardner, 2020, p. 73).

Researchers (e.g., Sweller et al., 1998; Zheng and Greenberg, 2018) point out that the goal of instructional design should focus on reducing extraneous cognitive load and optimizing germane cognitive load so the learner has sufficient cognitive resources to engage in meaningful knowledge construction. 


\section{Domain Prior Knowledge and Intrinsic Cognitive Load}

As previously mentioned, intrinsic cognitive load refers to the content difficulty and cannot be altered or changed. However, some researchers (e.g., Pollock, Chandler, \& Sweller., 2002) argue that content difficulty is relative to individuals' prior knowledge in a specific domain. A mathematics problem that is difficult for a novice learner may be less challenging for an experienced learner who has meaningful mathematical knowledge units stored in his or her long-term memory. Pollock et al. (2002) conducted a study to investigate the relationship between domain prior knowledge and intrinsic cognitive load in science. They employed a two-step instructional strategy by first providing the learners with isolated concepts of electric circuit (e.g., current, resistance) followed by more complex content related to the testing of electric current. Pollock et al. artificially isolated concepts, which, in turn, reduced learners' intrinsic cognitive load during learning, albeit an incomplete understanding of the problem by the learners. With the initial schema constructed, the learners were able to better deal with the problem presented in full complexity later on. Clarke, Ayres and Sweller (2005) investigated the role of intrinsic cognitive load and prior knowledge in mathematics learning. They found high level of intrinsic cognitive load would render learners incapable of learning new content. By implementing an instructional strategy called sequencing, they discovered the learners were able to build their prior knowledge, thus alleviating their intrinsic cognitive load in learning. Given the relationship between domain prior knowledge and intrinsic cognitive load, the variable of domain prior knowledge is included in this study to understand the connection among cognitive load, cognitive resources and learner performance in mathematical problem solving.

\section{Relationship between Domain Prior Knowledge and Instructional Strategy}

While studies (Clarke et al., 2005; Pollock et al., 2002) have shown the role of instructional strategy in prior knowledge construction, it remains unknown whether levels of domain prior knowledge (e.g., high and low) would affect the outcomes of instructional strategy. Kalyuga (2007) hypothesized that an instructional strategy that is beneficial for low-prior knowledge learners may become relatively inefficient for high-prior knowledge learners, or vice versa. Kalyuga, Chandler, and Sweller (1998) conducted a longitudinal study on the effect of instructional strategy (visual diagrams) on high- and low-prior knowledge learners in science learning. The novice learner initially studied the content with diagrams embedded in the text and scored well on the performance test. After a period of intensive training a reversal effect was observed: the same group of learners learned the content with diagrams only and demonstrated a high performance on the subsequent test. The authors explained that at the beginning novices lacked the adequate schemata to understand the complex content, therefore, the additional text explanation helped the novices comprehend the content. However, as the learners gained more knowledge, the additional text information became unnecessary to their learning. Kalyuga (2007) describes this phenomenon as expertise reversal effect. The expertise reversal effect principle advances Pollock's findings by revealing the interaction between domain prior knowledge and instructional strategy. The significance of this principle lies in its explanatory power of the role and function of domain prior knowledge in relation to instructional strategy. That is, the outcome of an instructional strategy is subject to the level of domain prior knowledge of the learner. The following section examines the function of worked example - an instructional strategy relevant to the current study and its relation to domain prior knowledge.

\section{Expertise Reversal Effect and Worked Example in Mathematics}

One of the widely studied and well applied instructional strategies in mathematics instruction is worked example (Große, 2015). As an instructional strategy, worked example facilitates schema construction and alleviate the mental load in learning since worked example demonstrates the problem-solving steps and solutions before learners try to solve the problems themselves (Richey \& Nokes-Malach, 2013; Saw, 2017; Yeo \& Tzeng, 2020). Worked example can be categorized into completion- and full-worked examples. Completion-worked example provides partial solution steps and expect the learners to finish the rest of the problem based on partial solution. Contrary to completion-worked example, full-worked example shows the entire steps of problem solving solution.

Sweller and Cooper (1985) examined the role of completion-worked example in complex mathematical problem solving. Two conditions were created: completion-worked example group and control group. The learners in completion-worked example condition were allowed to view half of the examples before they worked on the problems. The learners in control condition studied the problem without worked examples. Results showed that learners who studied with completion-worked examples outperformed those who did not $\left(U>30, \eta^{2}=.16\right)$. To further understand the effect of different types of worked examples on learning, Richey and Nokes-Malach (2013) studied learners' differences in deep understanding of the instructional materials between two types of worked examples: completion- and full-worked examples. In completion-worked example condition the learners were given worked examples with partial explanation and in the full-worked example condition the learners were provided with full explanation of the problems. What they found was that participants in the information 
withholding condition (i.e., completion-worked examples) demonstrated better conceptual learning and far transfer than participants in full explanation condition (i.e., full-worked examples) $\left(p<.01, \eta^{2}=.13\right)$. The authors argued that withholding instructional explanations may provide learners with an opportunity to engage in constructive learning activities to facilitate deeper learning and far transfer, whereas materials that include full explanations could suppress inference generation because the explanatory information was already present, thereby encouraging more passive learning activities such as rehearsal and paraphrasing. However, full-worked examples may be useful in schema construction as the step-by-step full explanations are effective in building knowledge blocks in longterm memory. Richey and Nokes-Malach thus concluded that the completion-worked examples facilitate constructive learning whereas the full-worked examples are conducive for schema construction. In the context of current study, the role of worked example is examined from the lens of domain prior knowledge. That is, it seeks to answer whether high- and low-prior knowledge learners would demonstrate the same level of engagement in constructive learning with completion-worked examples, or whether they would equally benefit from schema construction with full-worked examples.

While efforts have been made to investigate the differences in the types of worked examples, research that examines the interaction between domain prior knowledge and types of worked examples in mathematics is scarce, which has hampered the effective design and application of worked examples in education. As such, further research in domain prior knowledge and worked example is warranted. Based on Kalyuga's (2007) expertise reversal effect principle, it is hypothesized that low domain-prior-knowledge learners would benefit from fullworked examples as the strategy would facilitate novices' schema construction, whereas high domain-priorknowledge learners would perform well with completion-worked examples since they promote constructive thinking in learning (Richey \& Nokes-Malach, 2013).

\section{Task Difficulty}

Of particular interest to researchers is the relationship between instructional strategy and task difficulty (Lynch et al., 2019. Latta, 1978). Task difficulty refers to the task requirements defined by the level of content difficulty and associative cognitive demands. Latte (1978) conducted a study on learning orientation, feedback, and task difficulty (easy and difficult). The results showed a significant interaction between feedback and task difficulty $(p$ $<.01, \eta^{2}=.11$ ) with feedback responding differently to the level of task difficulty. It was found feedback had little impact on easy tasks but had positive effects on difficult tasks.

Evidence from preliminary studies further demonstrates that domain prior knowledge may play a role in the interaction between task difficulty and instructional strategy (Dhlamini, 2016; Orvis, Horn, \& Belanish, 2008). Orvis et al. (2008) examined the role of instructional strategy in video-game based learning. They found an interaction between instructional strategy and task difficulty with the forced adjustment strategy increasing task difficulty and the learner-centered adaptive adjustment strategy alleviating the task difficulty, which was largely explained by the learners' domain prior knowledge. That is, the task difficulty was mitigated due to an adaption of the content to the learner's domain prior knowledge. So far, the research on task difficulty in light of domain prior knowledge and instructional strategy has been focused on (a) the interaction between task difficulty and instructional strategy (Janning, Schatten, \& Schmidt-Thieme, 2016) and (b) the supplementary role of domain prior knowledge (Orvis et al., 2008). There is no study, to the best of our knowledge, that exploits the interaction between task difficulty and instructional strategy based on expertise reversal effect principle. Moreover, there have been mixed results regarding instructional strategy and task difficulty. Cevik and Altunt (2016) compared three instructional strategies (information only; information with demonstration; information with demonstration and application) in complex cognitive task performance and found no significant differences in group performances among three strategies in terms of task difficulty $(p>.05)$. Given the equivocal findings and a lack of research taken from expertise reversal effect, the current study examined the role of task difficulty in instructional strategy by putting in perspective the learners' expertise reversal effect to further understand the interaction among task difficulty, worked example and domain prior knowledge in mathematical problem solving.

\section{Motivation and Cognitive Load}

Research suggests that cognitive load, especially germane cognitive load is strongly correlated with motivational aspects of learning including science education (Um, Plass, Hayward, \& Homer, 2012). Researchers have been trying to find the psychometric correlation between the motivation measures and the types of cognitive load (Schnotz, Fries, \& Horz, 2009). Schnotz et al. (2009) compared Questionnaire of Current Motivation (QCM) developed by Rheinburg, Vollmeyer and Burns (2001) with Paas's (1992) Cognitive Load Measure (CLM) that measures overall cognitive load. They concluded that there was an overlap of probability of success between QCM and CLM. Recently, Leppink. Paas, van der Vleuten, van Gog, and van Merriënboer (2013) developed a ten-item questionnaire based on Paas's (1992) CLM. The instrument treats three types of cognitive load (e.g., intrinsic, 
extraneous and germane cognitive load) as distinct cognitive constructs in measurement that provides the opportunity to directly compare the motivation measures with each type of cognitive load, particularly the germane cognitive load in learning.

Notwithstanding the efforts to advance the understanding of the relationship between motivation and cognitive load, empirical research that focuses on motivation and germane cognitive load using specific measures like QCM and Leppink et al.'s CLM is lacking. Given the connection between germane load and motivation (Kirschner, 2002), an important endeavor of the current study was to verify the connection between germane cognitive load and motivation based on Leppink et al.'s CLM and Rheinbutg et al.'s QCM measures in mathematics problem solving.

\section{Research Questions}

Based on the literature, the following research questions were proposed as a basis to guide the current study:

Research Question 1: Is there a three-way interaction between prior knowledge, worked example and task difficulty as measured by posttest and three types of cognitive load?

Research Question 2: Are there interactions between (a) prior knowledge and worked example and (b) prior knowledge and task difficulty as measured by posttest and three types of cognitive load?

Research Question 3: Are there any correlations between three types of cognitive load defined in Leppink et al.'s CLM and the submeasures defined in Rheinbutg et al.'s QCM?

\section{The Study}

The present study considered the effects of domain prior knowledge, worked example, and task difficulty on learners' abilities to solve algebraic problems as measured by performance achievement and cognitive load. It also aimed to examine the relationship between three types of cognitive load and sub-dimensions of QCM measures. To understand the interaction between prior knowledge and worked example, two forms of worked examples were created: full- and completion-worked examples (Table 1). The full-worked examples provided the learners with solution directions and steps at each level whereas the completion-worked examples provided partial solutions with answers withheld at each step. It was predicted that the full-worked examples would benefit low priorknowledge learners' schema construction as the provision of full solution steps makes it easy for learners to understand and master the content. The completion-worked examples, on the other hand, would support high prior-knowledge learners constructive learning as withholding solutions steps promotes constructive cognitive thinking and processing (Richey \& Nokes-Malach, 2013).

Table 1. Samples of full- and completion-worked examples for simultaneous equation algebra problems

Full Worked Example
Solve the following simultaneous equations for variables $\mathrm{x}$ and $\mathrm{y}$ :
$\begin{aligned} & 12 \mathrm{x}+3 \mathrm{x}=45 \\ & 4 \mathrm{x}-5 \mathrm{y}=62\end{aligned}$
Solution: For this particular question, in order to solve for
variables $\mathrm{x}$ and $\mathrm{y}$, we first need to combine the like variables. We
can see that Eq 1 has all $\mathrm{x}$ variables. Therefore, they can be
combined.

\begin{tabular}{|c|c|}
\hline Objective 1: Combine $\mathrm{x}$-terms in $\mathrm{E}$ & \\
\hline Given from Eq 1 that & $12 x+3 x=45$ \\
\hline Add $\mathrm{x}$-terms as & $15 x=45$ \\
\hline Divide by 15 on both sides gives & $\mathrm{x}=(45 / 15)$ \\
\hline Simplify & $x=3$ \\
\hline Objective 2: $\underline{\text { Plug-in value of } \mathrm{x} \text { fr }}$ & $\mathrm{A}$ in $\mathrm{Eq} 2$ \\
\hline Given from Eq 2 that & $4 x-5 y=62$ \\
\hline Substitute value of $\mathrm{x}$ from $\mathrm{A}$ above & $4(3)-5 y=62$ \\
\hline Simplify & $\begin{array}{l}12-5 y=62 \\
-5 y=62-12 \\
-5 y=50\end{array}$ \\
\hline Divide by -5 & $y=-10$ \\
\hline
\end{tabular}

Answer: $\mathrm{x}=3 ; \mathrm{y}=-10$ Completion Worked Example

Solve the following simultaneous equations for variables $\mathrm{x}$ and $\mathrm{y}$ :

$$
\begin{aligned}
& 2 x+13 x=75 \\
& -x+2 y=5
\end{aligned}
$$

Solution: For this particular question, in order to solve for variables $x$ and $y$, we first need to combine the like variables. We can see that Eq 1 has all $x$-terms. Therefore, they can be combined.

Objective $1:$ Combine $\mathrm{x}$-terms in Eq 1
$\begin{aligned} & \text { Given from Eq } 1 \text { that } \\ & \text { Adding all } \mathrm{x} \text {-terms }\end{aligned}$
$\begin{array}{ll}\text { Dividing by the coefficient of } \mathrm{x} \text { on both sides } \\ \text { Simplify } & \mathrm{x}=(75 / \\ \mathrm{x} & =\end{array}$

Objecitve 2: Plug-in value of $\mathrm{x}$ in Eq 2 Given from Eq 2 that $\quad-x+2 y=5$

Substitute value of $\mathrm{x}$ from A above $-\ldots+2 \mathrm{y}=5$ Simplify

$2 \mathrm{y}=5+$

Divide by 2 on both sides

$2 \mathrm{y}=$ $\mathrm{y}=$

Answer: $\quad x=\ldots y=$ 


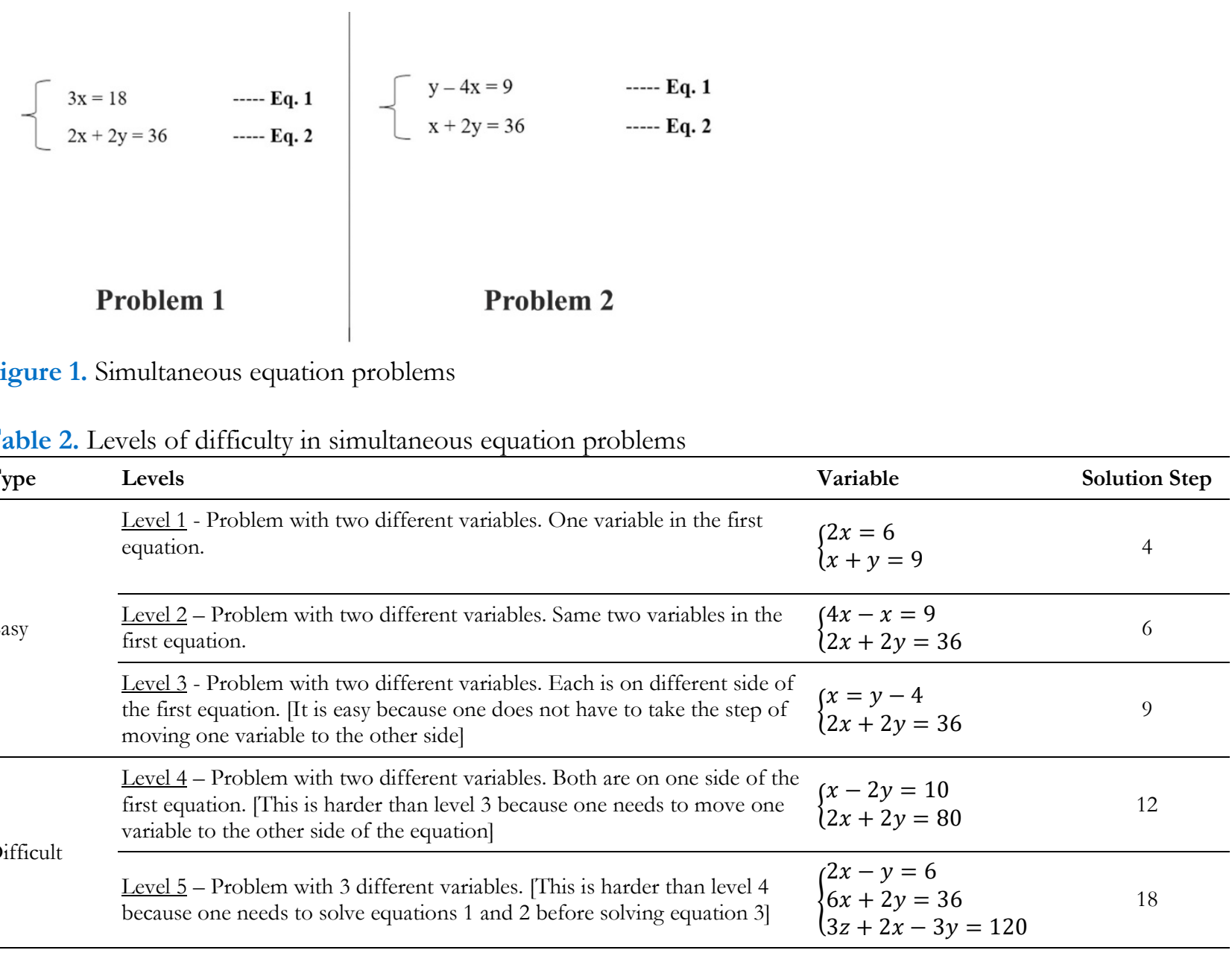

\section{Defining difficult and easy tasks}

The task difficulty is defined based on Sweller's (1988) production/solution step method. According to Sweller, the difficulty of the content, which is often considered a proxy for intrinsic cognitive load, is determined by the production/solution steps involved in problem solving or learning. Thus, the solution step method was used to define the difficult level of the problems. Five difficult levels were identified: Levels 1-3 were defined as easy problems since they contained fewer solution steps with one or two variables, whereas Levels 4-5 were defined as difficult because they involved more solution steps with more variables. Consider solving following two simultaneous equation problems (Figure 1). Problem 1 involves two different variables ( $\mathrm{x}$ and $\mathrm{y}$ ) and requires three solution steps. In solving Problem 1 the learner will (a) obtain the $x$ value in Eq.1 by dividing 3 on both sides of the equation $(3 x / 3=18 / 3)$, (b) replace the $x$ value in Eq. 2 with the $x$ value obtained in Eq. $1(2 * 6+2 y=36)$, and (3) calculate the value $y$.

In contrast, the solution steps become more complicated when solving Problem 2. There are several ways to solve Problem 2. Assume the problem can be solved by (a) finding the value of $y$ in Eq.1 $(y=9+4 x)$, (b) replacing the $y$ in Eq. 2 with the $y$ value obtained in Eq.1 $(\mathrm{x}+2(9+4 \mathrm{x})=36)$, (c) finding the $x$ value in Eq.2, and finally (d) substituting the $x$ value in Eq. 1 with the $x$ value obtained in Eq. 2 to find the $y$ value. Evidently, the solution to solving Problem 2 involves more steps and thus requires more mental effort than these in Problem 1. As such, the task of Problem 2 is considered more difficult compared to that of Problem 1. Table 2 shows the levels of task difficulty by variables and solution steps.

\section{Defining high-and low-prior knowledge learners}

Since domain prior knowledge was used as an independent variable to test the interaction between worked example, task difficulty and learner expertise, the issue of how to divide the prior knowledge variable into categorical data came to fore. Two different methods exist in regard to how to define high- and low-prior knowledge learners. They are: median split method and tri-split method. The median split method finds the median point and splits a continuous variable like prior knowledge into half (Aziz, Wuensch, \& Brandon, 2010; Iacobacci, Posavac, Kardes, Schneider, \& Popvich, 2015). The drawback of median split method is that it arbitrarily defines 
the participants who are one position above and below the median point as high- or low-prior knowledge learners which, as Liu and Reed (1994) point out, may significantly skew the outcomes. McClelland, Lynch, Irwin, Spiller, and Fitzsimons (2015) warned that median-split method is likely to increase Type II error (also see Rucker, McShane, \& Preacher, 2015). In contrast to median split method, Liu and Reed (1994) proposed a tri-split method that divided the participants into upper-third quarter, lower-third quarter and middle-third quarter. It eliminates the middle-third quarter and only keeps the upper- and lower- third quarters in its final analysis. Since the tri-split method eliminates middle one-third sample, it clearly creates the high and low categories by retaining top and bottom one-third samples, thus avoiding artificially labelling the samples as high or low and minimizing the risk of Type II error.

\section{METHOD}

\section{Participants}

One hundred and sixty participants were recruited from a Research I university in the western United States. Participants were non-science major college students enrolled in different academic programs. Of 160 participants, 114 participants earned credit towards their coursework, 46 participants received monetary compensation with an honorarium mentioning their participation in the study. The average age of participants was 23.25 with a standard deviation of 6.01. About 117 participants were females and 43 were males. Regarding ethnicity composition, 116 were whites, 19 were Hispanic, 13 were Asians, 1 was African-American and 11 were others. Participants were informed of the nature of the study and were asked to complete the consent process before participating. Participation in the study was voluntary. Approval from the appropriate Institutional Review Boards was obtained prior to beginning the study.

\section{Instrumentation}

The instruments consisted of (a) learning materials, (b) pretest, (c) posttest, (d) Cognitive Load Measurement (CLM), and (e) Questionnaire on Current Motivation (QCM).

Learning Materials. The learning materials (12 algebraic problems) were developed based on a middle school algebra textbook (McGraw Hills, Algebra-2) that covers topic of systems of equations. Problems on simultaneous equations were adapted for the study. Feedback from content experts and university faculty members was received with subsequent modification made to better meet the purpose of the study. A pilot study was run with the final problems reporting a high item reliability of Cronbach's Alpha $=.89$.

Two versions of problems were created: full- and completion-worked examples. The full-worked examples provided complete solution steps at every level; the completion-worked examples provided partial solution steps by withholding information in the problem solving process. The problems ranged from easy to difficult based on the variables and production steps involved in the problem solving (see Table 2).

Pretest. A 10-problem pretest was developed to understand learners' domain prior knowledge on the subject. Problems varied from easy and difficult with a maximum of 2.5 points for each problem. The total possible points to be obtained on the pretest was 25 points. Participants were required to solve the problems in the time span of 15 minutes. They were not only required to write down the answers but also to show all their work in solving pretest problems. The test results were graded by two independent graduate students showing an interrater reliability of Cronbach's Alpha $=0.993$.

Posttest. The posttest consisted of 10 problems on simultaneous equations. Like pretest, the problems varied in difficulty ranging from easy to difficult with a maximum of 2.5 points for each problem. The total possible points to be obtained on the pretest was 25 points. The test results were graded by two independent graduate students showing an interrater reliability of Cronbach's Alpha $=0.969$.

To ensure that difficulty level for the posttest was appropriate and met the purpose of the study, an additional item difficulty analysis was conducted. The biserial correlations showed a range between 0.22 and 0.64 for easy problems and a range between 0.31 and 0.52 for difficult problems, both of which were within the admissible range (Kavitha, Vijaya and Saraswathi, 2012) indicating the test items fit well with the purpose of the study.

CLM. To understand the impact of worked example, domain prior knowledge and task difficulty on cognitive load, the CLM (Leppink et al., 2013) was used. It is a 10-item self-report questionnaire with a Likert scale from 0 (Not at all the case) to 10 (Completely the case). The instrument measures three aspects of cognitive load: intrinsic (items 1-3), extraneous (items 4-6), and germane (items 7-10) cognitive load. Examples of intrinsic cognitive load measure would include: "The topic/topics covered in the activity was/were very complex", "The activity covered concepts and definitions that I perceived as very complex." The maximum possible points the participant could obtain on intrinsic load are 33. The examples of extraneous cognitive load measure would be: "The instructions and/or explanations during the activity were very unclear", "The instructions and/or explanations during the 
Table 3. A 2 × 2 × 2 Design for Prior Knowledge, Worked Example, and Task Difficulty

\begin{tabular}{lcccc}
\hline & \multicolumn{2}{c}{ Higher Prior Knowledge } & \multicolumn{2}{c}{ Lower Prior Knowledge } \\
\hline Full worked & Easy & Difficult & Easy & Difficult \\
examples & High PK / & High PK / & Lower PK / & Lower PK / \\
\hline $\begin{array}{l}\text { Completion worked } \\
\text { examples }\end{array}$ & Easy full WE & Difficult full WE & Easy full WE & Difficult full WE \\
\hline
\end{tabular}

activity were very unclear." The maximum possible points the participant could obtain on extraneous load are 33 . Finally, the examples of germane cognitive load measure would include: "The activity really enhanced my knowledge and understanding of the topic(s) covered", "The activity really enhanced my understanding of concepts and definitions." The maximum possible points the participant could obtain on germane load are 44. The instrument reported a consistent reliability with Cronbach alpha of .81 for intrinsic load, .75 for extraneous load, and .82 for germane load.

QCM. The QCM, developed by Rheinberg, Vollmeyer, and Burns (2001) is an 18-item self-report questionnaire with a 7-point Likert scale in which 1 means one agrees least to the statement and 7 means one agrees most to the statement. The possible maximum points one can obtain in QCM is 126 points. The instrument measures four constructs in motivation: interest, probability of success, anxiety, and challenge. For example, the statement "After reading the instruction, the task seems to be interesting to me" measures participants" interest, the statement of "I think everyone can manage this" probes probability of success, the statement of "If I think about this task, I feel a little bit worried" measures anxiety, and the statement of "I feel up to the difficulty of this task" measures challenge. The instrument reported high item reliability with Cronbach alpha .90 .

\section{Design of the Study}

The interaction between worked example (full- vs. completion-worked examples), prior knowledge (high vs. low) and task difficulty (easy vs. difficult) were studied with a $2 \times 2 \times 2$ factorial design. This allowed us to explore possible effects of full- and completion-worked examples in the context of domain prior knowledge and task difficulty and answer the question of to what extent domain prior knowledge and task difficulty may influence the outcomes of full- and completion-worked examples. Table 3 presents eight possible learning conditions to which high- and low-prior knowledge learners were randomly assigned.

\section{Procedure}

After signing the consent form, participants $(N=160)$ were asked to complete the pre-QCM which measured motivation in learning, followed by the pretest on simultaneous equation algebra. Based on the results of pretest, the participants were divided into high, low, and middle prior-knowledge levels with one-standard deviation above the mean as high-prior knowledge $(n=55, m=10.564, S D=1.808)$ and one-standard deviation below the mean as low-prior knowledge $(n=60, m=3.458, S D=1.808)$. The middle sample $(n=45, m=6.7, S D=3.3)$ was eliminated from final analysis, leaving 55 in the high-prior-knowledge group and 60 in the low-prior-knowledge group for final analysis.

Then the participants were randomly assigned to one of the eight learning conditions (Table 3). During the learning phase, the participants were asked to study the simultaneous equation algebraic content on a computer with either full- or completion-worked examples depending on the condition, followed by the practice of the problems in the booklet. The learning phase was self-paced but the maximum time to complete the session was one hour. Immediately upon completing the learning phase along with the practice booklet, participants were given the self-report CLM questionnaire to report their cognitive load on learning. Participants were then asked to complete a posttest on simultaneous equation. A post-QCM survey was also administered.

\section{Results}

This study explored the interaction between domain prior knowledge, worked example and task difficulty in algebraic problem solving. A $2 \times 2 \times 2$ one-way ANOVA was employed to analyze the three-way interaction. The independent variables included domain prior knowledge (high vs. low), worked example (full vs. completion worked examples) and task difficulty (easy vs. difficult) with post achievement test, CLM and post QCM scores as dependent variables. A family-wise alpha level of .05 was adopted for all analyses with p-values between .05 and .10 labelled as marginal effects and those greater than .10 as not significant. 
Table 4. Means and Standard Deviations for A 2 × 2 × 2 Design with Post-Achievement Test, Three Categories of Cognitive Load, and Post-QCM Scores

\begin{tabular}{|c|c|c|c|c|c|c|c|}
\hline Worked Examples & Expertise & Task Difficulty & Posttest & Intrinsic Load & Extraneous Load & Germane Load & $\begin{array}{l}\text { Post QCM } \\
\end{array}$ \\
\hline \multirow{4}{*}{ Completion WE } & \multirow{2}{*}{ Lower PK } & Difficult & $4.79(2.09)$ & $15.50(6.93)$ & $5.25(5.57)$ & $25.92(9.28)$ & $75.00(12.61)$ \\
\hline & & Easy & $5.94(2.70)$ & $12.59(7.45)$ & $3.06(3.59)$ & $25.06(12.09)$ & $75.41(14.76)$ \\
\hline & \multirow{2}{*}{ Higher PK } & Difficult & $11.19(2.06)$ & $11.08(5.64)$ & $9.15(8.18)$ & $13.23(9.00)$ & $67.46(14.21)$ \\
\hline & & Easy & $10.87(2.49)$ & $5.00(3.84)$ & $3.56(3.61)$ & $11.50(10.18)$ & $75.13(12.75)$ \\
\hline \multirow{4}{*}{ Full WE } & \multirow{2}{*}{ Lower PK } & Difficult & $6.68(2.95)$ & $15.44(7.50)$ & $8.31(7.19)$ & $24.06(10.93)$ & $74.00(18.09)$ \\
\hline & & Easy & $6.16(1.91)$ & $13.40(6.94)$ & $7.20(6.46)$ & $24.67(11.48)$ & $77.67(16.06)$ \\
\hline & \multirow{2}{*}{ Higher PK } & Difficult & $10.00(2.78)$ & $10.46(9.66)$ & $8.92(10.10)$ & $16.77(11.67)$ & $74.46(6.91)$ \\
\hline & & Easy & $12.00(2.38)$ & $6.08(6.88)$ & $2.92(2.95)$ & $23.46(11.28)$ & $73.77(8.42)$ \\
\hline
\end{tabular}

\section{Posttest}

Prior Knowledge * Worked Example * Task Difficulty
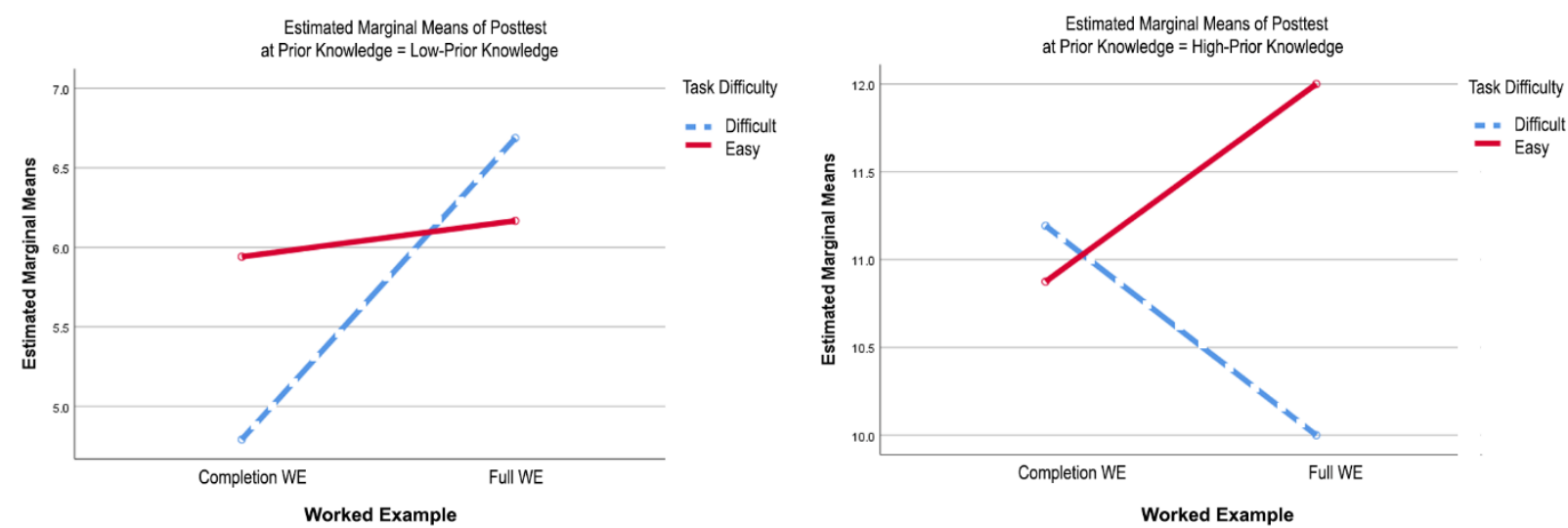

Figure 2. Three-way interaction between prior knowledge, worked example and task difficulty by posttest

The means and standard deviations for domain prior knowledge, worked example, and task difficulty with posttest, three categories of cognitive load, and post-QCM scores are presented in Table 4.

Research question 1 exploits the interaction between three independent variables in terms of (a) posttests and (b) types of cognitive load. There was a significant three-way between-subjects effects by posttest $\left(F_{1,107}=4.61, p\right.$ $<.05, \eta^{2}=.04$ ) (Figure 2), but no significant three-way interaction for types of cognitive load was detected. Further analyses revealed some interesting trends in terms of prior knowledge and task difficulty regarding types of cognitive load. None of the cognitive load was significant for worked example.

Prior knowledge. There were significant differences between high- and low-prior knowledge learners in terms of intrinsic $(F=22.424, p<.000)$ and germane cognitive load $(F=18.417, p<.000)$, but no significance was found between high- and low-prior knowledge learners for extraneous cognitive load $(F=.018, p=.895)$, suggesting the weak presence of extraneous cognitive load may have contributed to the active roles of intrinsic and germane cognitive loads in learning. In other words, with the influence of extraneous cognitive load abated, the cognitive resources in the working memory become available for the efforts to solve complex mathematics problems that were related to germane (efforts) and intrinsic (content complexity) cognitive load.

Task difficulty. Significant differences were found between difficult and easy tasks in terms of intrinsic $(F=$ $9.152, p<.001)$ and extraneous cognitive load $(F=10.761, p<.001)$, but no significance was found for germane cognitive load $(F=.204, p<.653)$. The results indicate high intrinsic or extraneous cognitive load due to task difficulty may render germane cognitive load unavailable for learning.

Regardless of the non-significance of three-way interaction by cognitive load, Research Question 1 was partially supported with a significant three-way interaction by posttest.

Research question 2 examines the interactions between (a) prior knowledge and worked example and (b) prior knowledge and task difficulty as measured by posttest and three types of cognitive load. The results are reported as follows.

Prior knowledge and worked examples. No significant differences were observed between prior knowledge and worked example as measured by intrinsic or extraneous cognitive load. However, a significant interaction by germane cognitive load $\left(F_{1,107}=4.72, p<.05, \eta^{2}=.04\right)$ was found between prior knowledge and worked example, suggesting germane cognitive load may serve as a valid indicator for the cognitive process of high- and low-prior knowledge learners when studying with worked examples. There was no significant interaction between prior knowledge and worked example by posttest. 
Table 5. Correlations between Intrinsic, Extraneous, Germane Cognitive Load and Motivation

\begin{tabular}{|c|c|c|c|c|c|c|c|}
\hline & 1 & 2 & 3 & 4 & 5 & 6 & 7 \\
\hline Intrinsic Load Total & 1 & $.311^{* *}$ & $.216^{* *}$ & $.324 * *$ & $-.299 * *$ & -.077 & $.221 * *$ \\
\hline Extraneous Load Total & & 1 & $-.264 * *$ & .136 & $-.191 *$ & -.046 & -.054 \\
\hline Germane Load Total & & & 1 & -.140 & .129 & $.264 * *$ & .008 \\
\hline QCM_Anxiety & & & & 1 & $-.322 * *$ & -.182 & $.304 * *$ \\
\hline QCM_Probability of Success & & & & & 1 & $.288^{* *}$ & $-.194 *$ \\
\hline QCM_Interest & & & & & & 1 & $.203^{* *}$ \\
\hline QCM_Challenge & & & & & & & 1 \\
\hline
\end{tabular}

The follow-up analysis revealed a relationship between prior knowledge and worked example. Lower-prior knowledge learners performed better on the posttest with full-worked examples than completion-worked examples $(t(1,26)=1.98, p=.05,2$-tailed $)$. In contrast, higher-prior knowledge learners demonstrated higher performance mean in completion-worked examples $(M=11.19, S D=2.06)$ than full-worked examples $(M=10.00, S D=2.78)$. However, the difference did not reach significant level $(p=.22, n s$.$) .$

Prior knowledge and task difficulty. No significant interaction was observed between prior knowledge and task difficulty. The follow-up analysis revealed significant main effects for high- and low-prior knowledge learners by posttest $\left(F_{1,107}=121.64, p<.001, \eta^{2}=.53\right)$, intrinsic cognitive load $\left(F_{1,107}=21.40, p<.001, \eta^{2}=.17\right)$, and germane cognitive load $\left(F_{1,107}=18.12, p<.001, \eta^{2}=.15\right)$. The analysis on the main effect of task difficulty was significant (Wilks' Lambda $\left.\lambda=.86, p<.05, \eta^{2}=.14\right)$ as measured by intrinsic $\left(F_{1,107}=8.60, p<.01, \eta^{2}=.07\right)$ and extraneous cognitive load $\left(F_{1,107}=9.92, p<.01, \eta^{2}=.09\right)$, suggesting task difficulty may play a significant role in determining the presence of intrinsic and extraneous cognitive load. The findings are consistent with the literature on prior knowledge, cognitive load and performance (Kalyuga, 2007; Kalyuga et al., 1998; Pollock et al., 2002).

To address research question 3, the correlation analyses were performed with three types of cognitive load (i.e., intrinsic, extraneous, and germane cognitive load) and subcategories of QCM (i.e., anxiety, probability of success, interest, and challenge). The results showed that germane cognitive load was significantly positively correlated with interest $(r=.264, p<.01)$ suggesting the connection between germane cognitive load and learners' interest in learning. It was found intrinsic cognitive load was positively correlated with anxiety $(r=.324, p<.01)$ and challenge $(r=.221, p<.01)$, but negatively correlated with probability of success $(r=-.299, p<.01)$, indicating learners' anxiety and the content challenge may negatively impact the success of learning. A negative correlation between extraneous cognitive load and germane cognitive load $(r=-.264, p<.01)$ was detected which confirms Sweller et al.'s (1998) hypothesis about the negative relationship between extraneous and germane cognitive load. Finally, extraneous cognitive load was found to correlate negatively with the probability of success $(r=-.191, p<.05)$ in QCM.

\section{DISCUSSION}

The current study investigated the relations between prior knowledge, worked example, and task difficulty. A three-way $2 \times 2 \times 2$ one-way ANOVA was performed. The following discussion of the results will be based on the three research questions proposed earlier.

\section{The Interaction between Prior Knowledge, Worked Example and Task Difficulty}

A significant three-way interaction was found by posttest suggesting high- and low-prior knowledge learners' performance can be significantly influenced by worked example like full- and completion-worked examples and the variances in task difficulty. The finding provides preliminary evidence on how prior knowledge, worked example and task difficulty interact with each other to influence the learners' performance in algebraic problem solving.

\section{The Interaction between (a) Prior Knowledge and Worked Example and (b) Prior Knowledge and Task Difficulty}

The analysis on the interaction between prior knowledge and worked example shed light on how full- and completion-worked examples may impact high- and low-prior knowledge learners' germane cognitive load in problem solving. That is, by applying the type of worked examples to individual learners based on their prior knowledge, learners are more likely to exert their efforts in learning. This is particularly true with low-prior knowledge learners who studied with full-worked examples and high-prior knowledge learners who studied with completion-worked examples. 
Although no significant interaction was found between prior knowledge and task difficulty, there were significant main effects for task difficulty by intrinsic cognitive load and extraneous cognitive load. This means task difficulty may play a significant role in determining the presence of intrinsic and extraneous cognitive load. Further, the significant main effects for prior knowledge indicated that high- and low-prior knowledge learners differed significantly in intrinsic cognitive load and germane cognitive load when solving complex algebraic problems with different worked examples suggesting an expertise reversal effect, which confirmed the findings in previous research (Kalyuga, 2007; Kalyuga et al., 1998; Pollock et al., 2002).

\section{Relationship between Cognitive Load and Motivation}

The study reported a positive correlation between germane cognitive load and interest confirming the connection between germane cognitive load and learners' interest as suggested by researchers in previous studies (Schnotz et al., 2009). The finding supports the germane cognitive load and interest correlation hypothesis showing that interest may be an important variable in designing effect instruction in learning.

The study further confirmed Sweller et al.'s hypothesis that extraneous cognitive load and germane cognitive load are negatively correlated, indicating higher extraneous cognitive load led to lower germane cognitive load. This finding has significant implications in STEM related education: in order to increase learners' efforts to learn (germane cognitive load), the educators must improve the design of instructional materials to lower the extraneous cognitive load by eliminating, for example, the redundancy or split-attention content in learning (Mayer \& Moreno, 2003). This is further supported by the findings of negative correlation between extraneous cognitive load and probability of success in the current study, suggesting with high extraneous cognitive load the learners would have few cognitive resources left in working memory, hence making little room for germane cognitive load in learning.

\section{CONCLUSIONS}

Complex learning like simultaneous equation problem solving can be cognitively demanding. While many factors (e.g., social, economic, cognitive, metacognitive, etc.) can influence learners' complex thinking, the current study focused on prior knowledge, worked example, and task difficulty from the perspectives of achievements, cognitive load and motivation in learning. By taking into perspective the interaction between prior knowledge, worked example and task difficulty, the current study revealed the relationship between learners' prior knowledge, worked examples, and task difficulty in complex learning in mathematics. It was found that lower-prior knowledge learners performed better with full-worked examples than completion-worked examples whereas higher-prior knowledge learners performed better with completion-worked examples than full-worked examples when solving complex math problems. The findings of the study also revealed that task difficulty may play a significant role in determining the presence of intrinsic and extraneous cognitive load. It is suggested that learners' intrinsic cognitive load can be largely explained by the difficulty of the tasks. Moreover, low-prior knowledge learners who experienced the task difficulty could undergo higher extraneous cognitive load if improper instructional strategy like completion-worked examples were used.

In addition to the findings regarding prior knowledge, worked example and task difficulty, the current study exploited the relationship between cognitive load and motivation. It was found that germane cognitive load is positively correlated with motivation as represented by interest in QCM. The findings provide empirical evidence showing the connection between germane cognitive load and interest suggesting motivational indicators like interest may be used as a proxy for germane cognitive load measure. The results also confirmed Sweller's (2018, Sweller et al., 1998) addictive hypothesis by showing the negative relationship of extraneous cognitive load with intrinsic and germane cognitive load. This finding is significant in that it guides the design of instruction in terms of cognitive load management in STEM learning.

\section{Implications of the Study}

As an important component of STEM (Science, Technology, Engineering and Mathematics), mathematics shares with science domains many cognitive and instructional processes in learning. The current study focuses on an important issue in STEM education: the management of cognitive resources in working memory for optimal learning. Taking from a cognitive load theory perspective, the study examines the relationship between learners' domain prior knowledge, task difficulty and worked example. While the domain of mathematics is used as the testbed in this proof-of-concept study, the findings are applicable to general science investigation and related instructional development since managing the cognitive resources in working memory by addressing the cognitive load is important to all learning including STEM education. As such, the findings of the current study have contributed to the research and practicing communities the understanding of the relationship between cognitive load and learning in light of learner prior knowledge, worked example and task difficulty. 


\section{Limitations of the Study}

Like any empirical studies, the current study is not without limitations. The failure to obtain a significant threeway interaction by cognitive load indicates that more work is needed to help learners understand the types of cognitive load when responding to the questionnaire. Sweller (2018) expressed similar concern by arguing that learners may not be familiar with the categories of cognitive load. Another limitation is the power of the study. More participants may be needed, especially the current study used the tri-split methods in defining high- and lowprior knowledge learners which could weaken the power of the study.

The current study explored the immediate effects of worked examples in relation to prior knowledge and task difficulty. Future research may examine the long-term effect by inviting participants back after a period of time to study learners' knowledge retention and transfer. A large sample size may be needed in future study. Studies in future may include online cognitive load measures like eye-tracking, dual-task, etc. to better understand the dynamic changes of cognitive load including peak load, cumulative load, and overall load in complex learning.

\section{REFERENCES}

Ayres, P. (2018). Subjective measures of cognitive load: What can they reliably measure? In R. Zheng (Ed.), Cognitive load measurement and application: A theoretical framework for meaningful research and practice (pp. 9-28). New York: Routledge. https://doi.org/10.4324/9781315296258-2

Aziz, S., Wuensch, K., \& Brandon, H. (2010). A comparison among worker types using a composites approach and median splits. Psychological Record, 60(4), 627-641. https://doi.org/10.1007/BF03395736

Baddeley, A. (1990). Human memory: Theory and practice. Boston: Allyn and Bacon.

Baddeley, A. D., Hitch, G. (1974). Working Memory. Psychology of Learning and Motivation, 8, 47-89. https://doi.org/10.1016/S0079-7421(08)60452-1

Cevik, V., \& Altunt, A. (2016). Roles of working memory performance and instructional strategy in complex cognitive task performance. Journal of Computer Assisted Learning, 32, 594-606. https://doi.org/10.1111/jcal.12156

Clarke, T., Ayres, P., \& Sweller, J. (2005). The impact of sequencing and prior knowledge on learning mathematics through spreadsheet applications. Educational Technology Research \& Development, 53(3), 15-24. https://doi.org/10.1007/BF02504794

Cook, A., Zheng, R., \& Blaz, J. (2009). Measurement of cognitive load during multimedia learning activities. In R. Zheng (Ed.), Cognitive effects of multimedia learning (pp. 34-50). Hershey, PA: IGI Global. https:// doi.org/10.4018/978-1-60566-158-2.ch003

Dhlamini, J. (2016). Enhancing learners' problem solving performance in mathematics: A cognitive load perspective. European Journal of STEM Education, 1(1), 27-36. https://doi.org/10.20897/lectito.201604

Fuchs, L., Fuchs, D., \& Seethaler, P. M. (2020). Addressing the role of working memory in mathematical wordproblem solving when designing intervention for struggling learners. The International Journal on Mathematics Education, 52(1), 87-96. https://doi.org/10.1007/s11858-019-01070-8

Große, C. S. (2015). Fostering modeling competencies: Benefits of worked examples, problems to be solved, and fading procedures. European Journal of Science and Mathematics Education, 3(4), 364-375.

Gupta, U. (2015). Managing cognitive load during complex learning: A study on worked examples and element interactivity. Unpublished doctoral dissertation, University of Utah, Salt Lake City.

Hosbein, K. N., \& Barbera, J. (2020). Alignment of theoretically grounded constructs for the measurement of science and chemistry identity. Chemistry Education Research and Practice, 21(1), 371-386. https://doi.org/10.1039/C9RP00193J

Janning, R., Schatten, C., \& Schmidt-Thieme, L. (2016). Perceived task-difficulty recognition from log-file information for the use in adaptive intelligence tutoring systems. International Journal of Artificial Intelligence in Education, 26(3), 855-876. https:// doi.org/10.1007/s40593-016-0097-9

Kalyuga, S. (2007). Expertise reversal effect and its implications for learner-tailored instruction. Educational Psychology Review, 19, 509-539. https:/ / doi.org/10.1007/s10648-007-9054-3

Kalyuga, S., Chandler, P., \& Sweller, J. (1998). Levels of expertise and instructional design. Human Factors, 40, 1-17. https://doi.org/10.1518/001872098779480587

Kavitha, R, Vijaya, A., \& Saraswathi, D. (2012). Intelligent Item Assigning for Classified Learners in ITS using Item Response Theory and Point Biserial Correlation. 2012 International Conference on Computer Communication and Informatics, 1-5. https://doi.org/10.1109/ICCCI.2012.6158813

Kirschner, P. A. (2002). Cognitive load theory: implications of cognitive load theory on the design of learning. Learning and Instruction, 12(1), 1-10. https:/ / doi.org/10.1016/S0959-4752(01)00014-7 
Latta, R. M. (1978). Interactive effects of initial achievement orientation and prior success feedback on the mastery of subsequent difficult and easy tasks. American Educational Research Journal, 15, 17-24. https://doi.org/10.3102/00028312015001017

Lee, H., Plass, J., \& Homer, B. (2006). Optimizing cognitive load for learning from computer-based science simulations. Journal of Educational Psychology, 98(4), 902-913. https:/ / doi.org/10.1037/0022-0663.98.4.902

Leppink, J., Paas, F., van der Vleuten, C. P. M., van Gog, T., \& van Merriënboer, J. J. G. (2013). Development of an instrument for measuring different types of cognitive load. Behavior Research Methods, 45(4), 1058-1072. https://doi.org/10.3758/s13428-013-0334-1

Liu, M., \& Reed, W. M. (1994). The relationship between the learning strategies and learning styles in a hypermedia environment. Computers in Human Behavior, 10(4), 419-434. https:// doi.org/10.1016/0747-5632(94)90038-8

Liu, M., Toprac, P., \& Yuen, T. (2009). What factors make a multimedia learning environment engaging: A case study. In R. Zheng (Ed.), Cognitive effects of multimedia learning (pp. 173-192). Hershey, PA: IGI Global Publishing. https://doi.org/10.4018/978-1-60566-158-2.ch010

Lynch, R., Hurley, A., \& Cumiskey, O. (2019). Exploring the relationship between homework task difficulty, student engagement and performance. Irish Educational Studies, 38(1), 89-103. https://doi.org/10.1080/03323315.2018.1512889

Mayer, R. \& Moreno, R. (2003). Nine ways to reduce cognitive load in multimedia learning. Educational Psychologist, 38(1), 43-52. https://doi.org/10.1207/S15326985EP3801_6

McClelland, G., Lynch, J., Irwin, J., Spiller, S., \& Fitzsimons, G. (2015). Median splits, type II errors, and falsepositive consumer psychology: Don't fight the power. Journal of Consumer Psychology, 25(4), 679-689. https://doi.org/10.1016/j.jcps.2015.05.006

O'Conner, E., \& Domingo, J. (2020). Designing for immersion: Mindful of cognition, motivation, and imagination. In R. Zheng (Ed.), Cognitive and affective perspectives on immersive technology in education (pp. 22-50). Hershey, PA: IGI Global. https://doi.org/10.4018/978-1-7998-3250-8.ch002

Orvis, K. A., Horn, D. B., \& Belanish, J. (2008). The roles of task difficulty and prior videogame experience on performance and motivation in instructional videogames. Computers in Human Behavior, 24, 2415-2433. https://doi.org/10.1016/j.chb.2008.02.016

Paas, F. (1992). Training Strategies for Attaining Transfer of Problem-Solving Skill in Statistics: A Cognitive Load Approach. Journal of Educational Psychology, 84(4), 429-434. https:/ / doi.org/10.1037/0022-0663.84.4.429

Plass, J., \& Kalyguga, S. (2019). Four ways of considering emotion in cognitive load theory. Educational Psychology Review, 31(2), 339-359. https://doi.org/10.1007/s10648-019-09473-5

Pollock, E., Chandler, P., \& Sweller, J. (2002). Assimilating complex information. Learning and Instruction, 12, 61-86. https://doi.org/10.1016/S0959-4752(01)00016-0

Rheinberg, F., Vollmeyer, R., \& Burns, B. D. (2001). FAM: Ein Fragebogen zur Erfassung aktueller Motivation in Lern- und Leistungssituationen. Diagnostica, 47(2), 57-66. https://doi.org/10.1026/ /0012-1924.47.2.57

Richey, J. E., \& Nokes-Malch, T. J. (2013). How much is too much? learning and motivation effects of adding instructional explanations to worked example. Learning and Instruction, 25, 104-124. https://doi.org/10.1016/j.learninstruc.2012.11.006

Richter, J., \& Scheiter, K. (2019). Studying the expertise reversal of the multimedia signaling effect at a process level: Evidence from eye tracking. Instructional Science, 47(6), 627-658. https://doi.org/10.1007/s11251-01909492-3

Rucker, D., McShane, B., \& Preacher, K. (2015). A researcher's guide to regression, discretization, and median splits of continuous variables. Journal of Consumer Psychology, 25(4), 666-678. https://doi.org/10.1016/j.jcps.2015.04.004

Saw, K. G. (2017). Cognitive load theory and the use of worked examples as an instructional strategy in physics for distance learners: A preliminary study. Turkish Online Journal of Distance Education, 18(4), 142-159. https://doi.org/10.17718/tojde.340405

Schnotz, W. (2010). Reanalyzing the expertise reversal effect. Instructional Science, 38, 315-323. https://doi.org/10.1007/s11251-009-9104-y

Schnotz, W., Fries, S., \& Horz, H. (2009). Motivational aspects of cognitive load theory. In M. Wosnitza, S. A. Karabenick, A. Efklides, \& P. Nenniger (Eds.), Contemporary motivation research: From global to local perspectives, 69-96.

Smith, D., Zheng, R., Metz, A. J., Morrow, S., Pompa, J., Hill, J., \& Rupper, R. (2019). Role of cognitive prompts in video caregiving training for older adults: Optimizing deep and surface learning. Educational Gerontology, 45(1), 45-56. https://doi.org/10.1080/03601277.2019.1580442

Sweller J., \& Chandler P. (1991). Evidence for Cognitive Load Theory. Cognition and Instruction, 8, 351-362. https://doi.org/10.1207/s1532690xci0804_5 
Sweller, J. (2010). Element interactivity and intrinsic, extraneous, and germane cognitive load. Educational Psychological Review, 22, 123-138. https://doi.org/10.1007/s10648-010-9128-5

Sweller, J. (2018). The role of independent measures of load in cognitive load theory. In R. Zheng (Ed.), Cognitive load measurement and application: A theoretical framework for meaningful research and practice (pp. 1-8). New York, Routledge. https://doi.org/10.4324/9781315296258-1

Sweller, J., \& Cooper, G. A. (1985). The use of worked example as a substitute for problem solving in learning algebra. Cognition and Instruction, 12(1), 59-89. https://doi.org/10.1207/s1532690xci0201_3

Sweller, J., Van Merriënboer, J. J., \& Paas, F. (1998). Cognitive architecture and instructional design. Educational Psychology Review, 10(3), 251-296. https://doi.org/10.1023/A:1022193728205

Um, E. R., Plass, J. L., Hayward, E. O., \& Homer, B. D. (2012). Emotional design in multimedia learning. Journal of Educational Psychology, 104, 485-498. https://doi.org/10.1037/a0026609

Vygotsky, L. S. (1978). Mind in society: The development of higher psychological processes. Cambridge, MA: Harvard University Press.

Wallen, E., Plass, J., \& Brunken, R. (2005). The function of annotations in the comprehension of scientific texts: Cognitive load effects and the impact of verbal ability. Educational Technology Research and Development, 53(3), 59-72. https://doi.org/10.1007/BF02504798

Yeo, L. M., \& Tzeng, Y. T. (2020). Cognitive effect of tracing gesture in the learning from mathematics worked examples. International Journal of Science and Mathematics Education, 18(4), 733-751. https://doi.org/10.1007/s10763-019-09987-y

Zheng, R., \& Gardner, M. (2020). Memory in education. New York: Routledge.

Zheng, R., \& Greenberg, K. (2018). The boundary of different approaches in cognitive load measurement: Strengths and limitations. In R. Zheng (Ed.), Cognitive load measurement and application: A theoretical framework. for meaningful research and practice (pp. 45-56). New York, Routledge. https:// doi.org/10.4324/9781315296258-4 